\title{
Fluid Dynamics Android Application: An Efficient Semi-implicit Solver for Compressible Flows
}

\author{
Shivam Singhal ${ }^{\dagger 1}$, Yayati Gupta ${ }^{2}$, and Ashish Garg*†3 \\ ${ }^{1}$ Department of Mechanical Engineering, Indian Institute of Technology Kharagpur \\ ${ }^{2}$ Department of Computer Science \& Engineering, Indian Institute of Information Technology Dharwad \\ ${ }^{3}$ GATE Aerospace Forum Educational Services, Delhi-110059
}

\begin{abstract}
The computing power of smartphones has not received considerable attention in the mainstream education system. Most of the education-oriented smartphone applications (apps) are limited to general purpose services like Massive Open Online Courses (MOOCs), language learning, and calculators (performing basic mathematical calculations). Greater potential of smartphones lies in educators and researchers developing their customized apps for learners in highly specific domains. In line with this, we present Fluid Dynamics, a highly accurate Android application for measuring flow properties in compressible flows. This app can determine properties across the stationary normal and oblique shock, moving normal shock and across Prandtl - Meyer expansion fan. This app can also measure isentropic flows, Fanno flows, and Rayleigh flows. The functionality of this app is also extended to calculate properties in the atmosphere by assuming the International Standard Atmosphere (ISA) relations and also flows across the Pitot tube. Such measurements are complicated and time-consuming since the relations are implicit and hence require the use of numerical methods, which give rise to repetitive calculations. The app is an efficient semi-implicit solver for gas dynamics formulations and uses underlying numerical methods for the computations in a graphical user interface (GUI), thereby easing and quickening the learning of concerned users. The app is designed for the Android operating system, the most ubiquitous and capable surveillance platform, and its calculations are based on JAVA based code methodology. In order to check its accuracy, the app's results are validated against the existing data given in the literature.
\end{abstract}

Keywords: Fluid dynamics Android app, compressible flows, isentropic flows, Android app for gas dynamics.

\section{Nomenclature}

$\begin{array}{llll}* & \text { At sonic condition } & M & \text { Mach number } \\ A & \text { Area at cross-section }\left[\mathrm{m}^{2}\right] & P & \text { Static pressure }[\mathrm{Pa}] \\ A^{*} & \text { Throat area at choked condition in } & P_{o} & \text { Stagnation pressure }[\mathrm{Pa}] \\ & \text { convergent-divergent }(\mathrm{CD}) \text { nozzle }\left[\mathrm{m}^{2}\right] & R & \text { Local gas constant }\left[\mathrm{J} \mathrm{kg}^{-1} \mathrm{~K}^{-1}\right] \\ D & \text { Hydraulic diameter }[\mathrm{m}] & T & \text { Static temperature }[\mathrm{K}] \\ F & \text { Fanning friction factor } & T_{o} & \text { Stagnation temperature }[\mathrm{K}] \\ L^{*} & \text { Required duct length }[\mathrm{m}] & U & \text { flow speed }[\mathrm{m} / \mathrm{s}]\end{array}$

${ }^{*}$ Corresponding author, email: ashish@gateaerospaceforum.com,

${ }^{\dagger}$ S.S. and A.G. are combined first authors.

October 26, 2019 
$\gamma \quad$ Heat capacity ratio

$\mu \quad$ Mach angle [degree]

$\nu \quad$ Prandlt-Meyer function [degree]

$\rho \quad$ Static density $\left[\mathrm{kg} / \mathrm{m}^{3}\right]$

$\rho_{o} \quad$ Stagnation density $\left[\mathrm{kg} / \mathrm{m}^{3}\right]$

$\theta \quad$ Deflection Angle [degree]

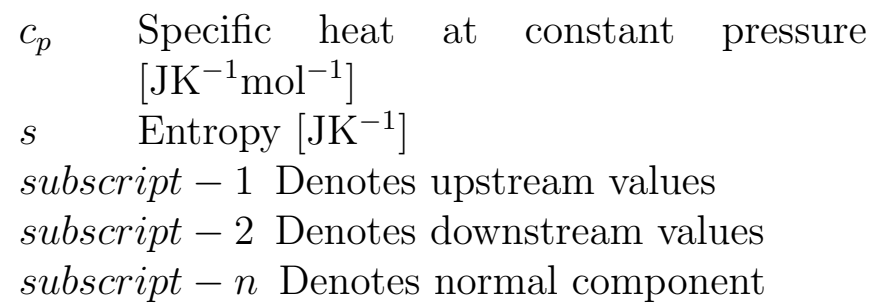

\section{Introduction}

In 2013, the engineering director of Ames research center, Pete Klupar, was thinking aloud in a speech; why the smartphones, which had faster processors and better sensors than many satellites were so cheap in comparison. The talk ignited the mind of Chris Boshuizen, a fellow researcher. By September 2013, Chris and his team at NASA launched three coffee-cup-size PhoneSats in orbit to beam back information. Interestingly, these devices were the ordinary Nexus phones with Android operating system (OS). NASA now has tiny satellites with smartphone brains. These phones wrap increasingly powerful technology in decreasingly smaller packages. No wonder, these small personal portable computers [1-4] are now killing desktops and laptops in terms of speed, cost, and convenience. 2.71 billion smartphone users [5] in the world generate more than half of the Internet's traffic [6]. $46 \%$ of these users report not being able to imagine their life without a phone [7]. Instead, the number of smartphones in the world is growing approximately five times faster than the world's population [8].

The dominant use of smartphones in the world seems restricted to general-purpose applications (apps) like Facebook, WhatsApp, YouTube, etc. However, digital trends now show a significant impact of smartphones on the educational system as well. The Apple iTunes store alone has more than 65,200 educational apps. Collectively, there are more than 500,000 educational apps developed to date. The most popular of these are Amazon Kindle (online book store), Coursera (provides Massive Open Online Courses), Duolingo (language learning app) and Photomath (all about math and equation solving). These apps enable millennial teachers to reach millions of digital learners. Hence, mobile learning is overhauling the traditional classroom setting of "chalk and talk" learning. Unlike traditional classrooms, mobile learning is available $24 \times 7$ at any place and pace. It is interactive, portable, and fun. Further, these apps enable immediate feedback, collaborative setting, and personalized learning.

Although mLearning apps have come a long way, most of them are designed not by the domain experts, rather by the software developers possessing advanced programming knowledge. This results in most of such apps being generic and lacking customization according to the subject of interest. This limits the diversity of experiences educators can provide to digital learners. It would be useful if educators could create their own apps as per their requirements; comprising tests, interactive maps, mobile features (e.g., GPS), etc. However, most educators lack the necessary skills required for developing such apps. This remains a significant gap between mobile technologies and their incorporation in education.

In this paper, we attempt to address this gap in a specific domain by developing a customized app to solve gas dynamics formulations. Gas dynamics utilizes the principles of mechanics, thermodynamics, aerodynamics and chemical kinetics to study the motion of compressible flows, particularly gases 9 11. Gas dynamics formulations play a significant role in the study of gases flowing at speed close to that of sound. Such formulations are applicable in the design of aircrafts, 
spacecrafts and their propulsion systems. Researchers have also addressed the compressible flow problems in supersonic flights and internal flows [9, 12]. However, solving these formulations requires one to carry out lengthy and repetitive calculations based on numerical methods like Newton Raphson and bisection method. We provide an easy to use graphical user interface (in the form of an Android app) to the users which comes in handy while solving these equations.

The major contributions of our study are

1. the development and presentation of an Android app for solving gas dynamics formulations for isentropic and non-isentropic flows which include:

- Isentropic relations

- Prandtl- Meyer expansion fan relation

- Normal shock properties

- Oblique shock properties

- Fanno flow relations

- Rayleigh flow relations

- International Standard Atmosphere (ISA) relations

- Moving normal shock relations

- Pitot tube relations

2. validation of the app's output with the data available in the literature.

\section{$1.1 \quad$ Related Applications}

There are several apps related to fluid mechanics in GooglePlay ${ }^{T M}$ repository https://play . google.com). Some of these apps are listed below for the reader's reference.

1. Fluid Flow Navier-Stokes - This app allows simulation of fluid flow based on Navier-Stokes equations. It allows the user to create fluids, simulate solar explosions and move a ball in a wind tunnel.

2. Liquid Simulation - It is an interactive simulation of the $2 \mathrm{D}$ incompressible viscous fluid model.

3. Hydraulic Calculator. This app calculates the pressure drop in the design of liquid, gas, and two-phase fluid pipelines taking into account the inline fittings.

4. Liquid Flow Calculation - It calculates flow rate through a pipe.

5. Open Flow - This app allows the user to calculate a channel's hydraulic properties such as flow capacity, flow width, flow depth, channel slope, Froude number, etc.

To the best of our knowledge, these existing apps can not measure the flow properties for gas dynamics formulations. Therefore we develop this app which can measure such flow properties, with an absolute error of $10^{-6}$. It can be downloaded from the GATE Aerospace Forum Educational Services repository under the name 'Fluid Dynamics App' 13. We use Eclipse JAVA integrated 
development environment (IDE) in developing this app. The app is portable and can run on any platform bearing the Android Operating system, e.g., a tablet or a smartphone.

The remaining paper is organized as follows. Section 2 presents the GUI design of the app with a number of snapshots. Section 3 provides an overview of gas dynamics formulations used for calculating the compressible flows in this app, along with corresponding schematic diagrams. The numerical methods for solving these formulations are reported in Section 4. Section 5 shows the validation of the calculated app results with the reference data available in the literature. The paper is concluded in Section 6.

\section{Fluid Dynamics' User Interface (UI) Design}

Figures 11 to 3 correspond to the user interface design of the app. After registration, the user is presented with a screen, as shown in Figure 1 .

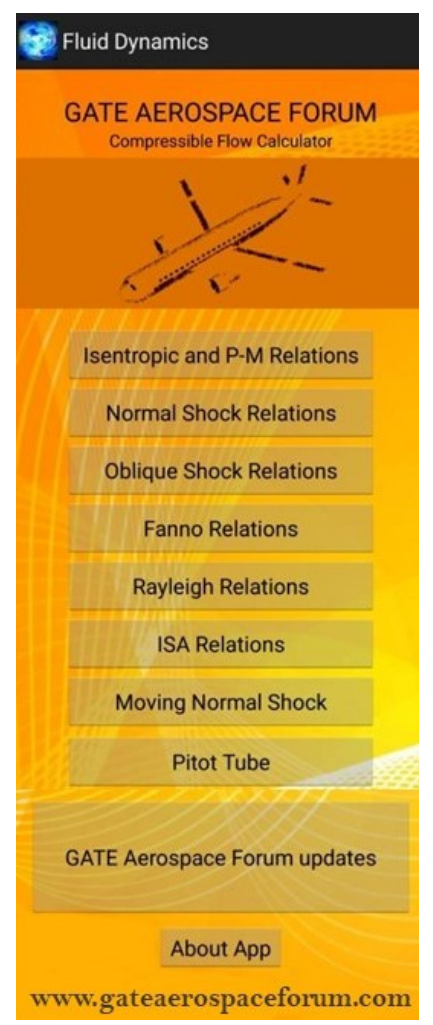

Figure 1: Snapshot of the screen showing the available formulations.

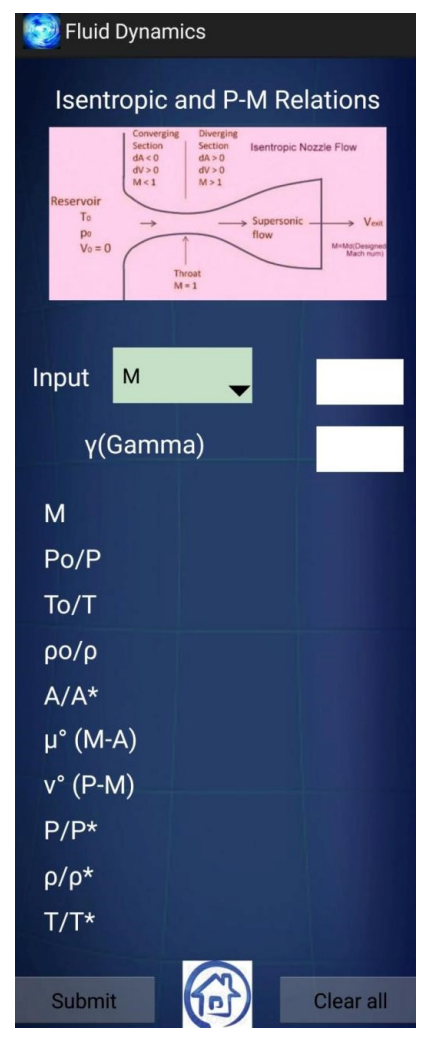

Figure 2: Snapshot of the screen after selecting the desired formulation ("isentropic and PM relations" in this case).

The screen shows a variety of formulations, any of which can be selected. Once a formulation is selected, the corresponding layout is presented to the user. For instance, if the user selected "isentropic and PM relations", then the corresponding layout is as shown in Figure 2, The layout comprises a drop-down list for receiving the inputs from the user (Figure 3a). Any of the known input can be used to solve the complete formulation. The app also allows the user to input the desired value of specific heat constant $(1 \leq \gamma \leq 1.67)$, depending on degrees of freedom of a molecule. After entering the desired inputs, the user can click on "Submit" button to see the results. As an instance, if the user selects ' $\mathrm{M}=5$ ' and ' $\gamma=1.5$ ', and then presses "Submit" 


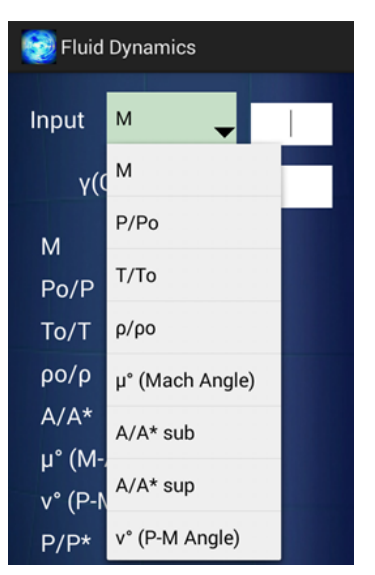

(a) Snapshot of possible inputs for "isentropic \& PM relations".

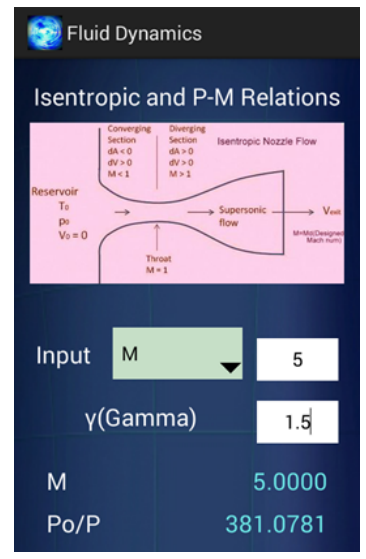

(b) Snapshot when input $\mathrm{M}=5$ and $\gamma=1.5$ are selected.

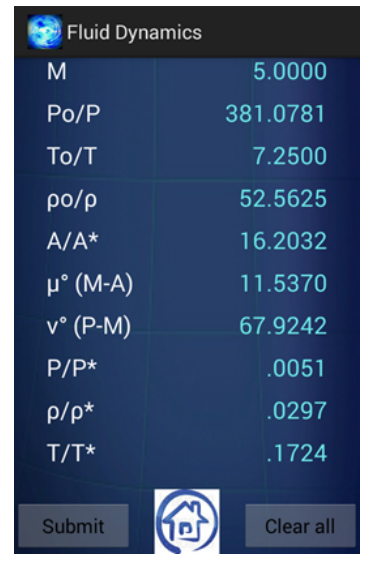

(c) Snapshot showing the output corresponding to Figure $3 \mathrm{~b}$

Figure 3: Series of snapshots depicting complete representation (from showing wide range of possible inputs to obtaining output data of "isentropic and PM relations") for input $\mathrm{M}=5$ and $\gamma=1.5$.

button (as shown in Figure 2), outputs as shown in Figures $3 \mathrm{~b}$ and $3 \mathrm{c}$ respectively are presented to her. The user interface layout of the app is explained with the help of a flow chart in Figure 4.

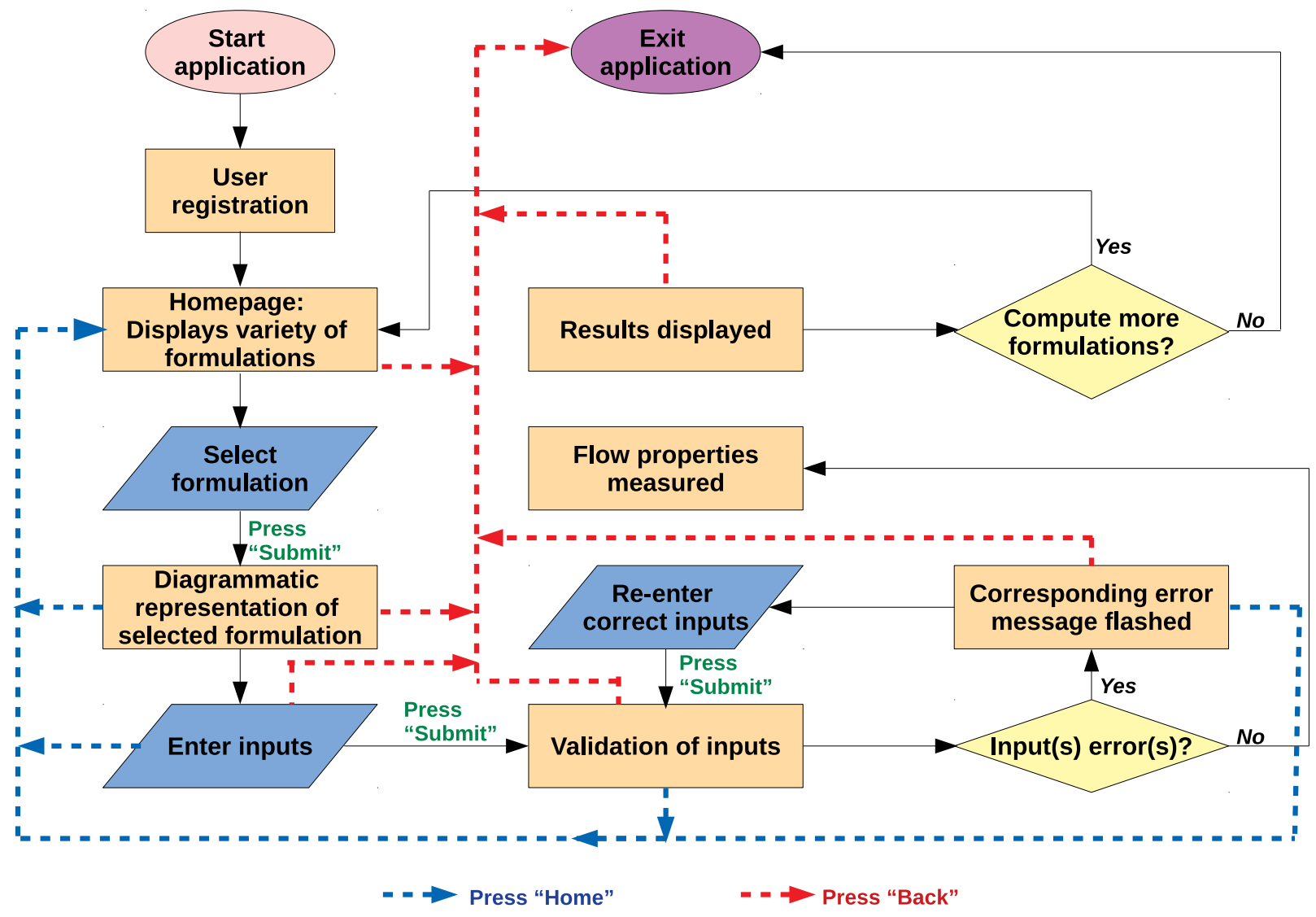

Figure 4: Flowchart representing the user interface layout of the "Fluid Dynamics" App. 


\section{Compressible Flows}

In this section, we present an overview of gas dynamics formulations for pressure, temperature, and density ratios as a function of Mach number $M$, specific heat constant ratio $\gamma$, and local gas constant $R$ (Please refer [10] for the derivations of these formulations). We solve these formulations in this app using JAVA based code programming for Android platform.

\subsection{Isentropic Relations}

Typically isentropic flows occur when the changes in the flow variables are small and gradual, leading to a reversible flow. A schematic diagram of the isentropic flow through a nozzle is shown in Figure 5. Equations (1) to (5) represent the isentropic relations between stagnation and static properties.

$$
\begin{gathered}
\frac{P_{o}}{P}=\left(\frac{\rho_{o}}{\rho}\right)^{\gamma}=\left(\frac{T_{o}}{T}\right)^{\gamma / \gamma-1} \\
\frac{T_{o}}{T}=\left(1+\frac{\gamma-1}{2} M^{2}\right) \\
\frac{P_{o}}{P}=\left(1+\frac{\gamma-1}{2} M^{2}\right)^{\frac{\gamma}{\gamma-1}} \\
\frac{\rho_{o}}{\rho}=\left(1+\frac{\gamma-1}{2} M^{2}\right)^{\frac{1}{\gamma-1}} \\
\left(\frac{A}{A^{*}}\right)^{2}=\frac{1}{M^{2}}\left[\frac{2}{\gamma+1}\left(1+\frac{\gamma-1}{2} M^{2}\right)\right]^{\frac{(\gamma+1)}{(\gamma-1)}} .
\end{gathered}
$$

These relations are derived using the energy conservation principle. As stated earlier, Figure $3 \mathrm{a}$ represents the user interface of the app. Here, the user can provide possible inputs for the isentropic and PM relations based on equations (1) to (5). For sonic condition, i.e., when $\mathrm{M}=1$,

$$
\frac{T_{o}}{T}=\frac{T_{o}}{T^{*}}, \frac{P_{o}}{P}=\frac{P_{o}}{P^{*}}, \frac{\rho_{o}}{\rho}=\frac{\rho_{o}}{\rho^{*}} .
$$

\subsection{Prandtl- Meyer Expansion Fan Relation}

In the expansion process of supersonic flows, a finite change in the flow direction generates PrandtlMeyer central expansion fan. Across the expansion fan, the flow diverts away from itself. This is an isentropic process. This expansion fan consists of an infinite number of Mach lines. Equations (7) and (8) show the first Mach line and the last Mach line for the initial flow direction and the final flow direction respectively. This has been shown in Figure 6 .

$$
\mu_{1}(M)=\sin ^{-1} \frac{1}{M_{1}}
$$




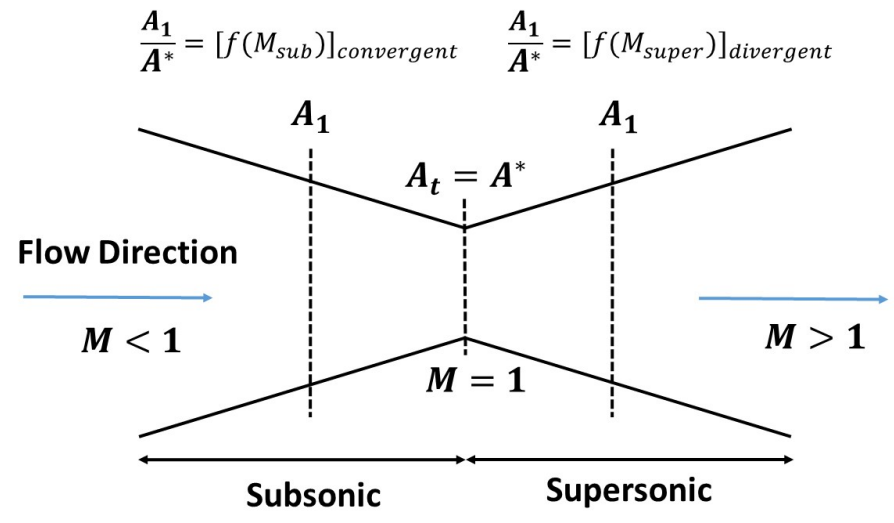

Figure 5: Schematic diagram representing ideal flow through convergent-divergent nozzle at choked condition.

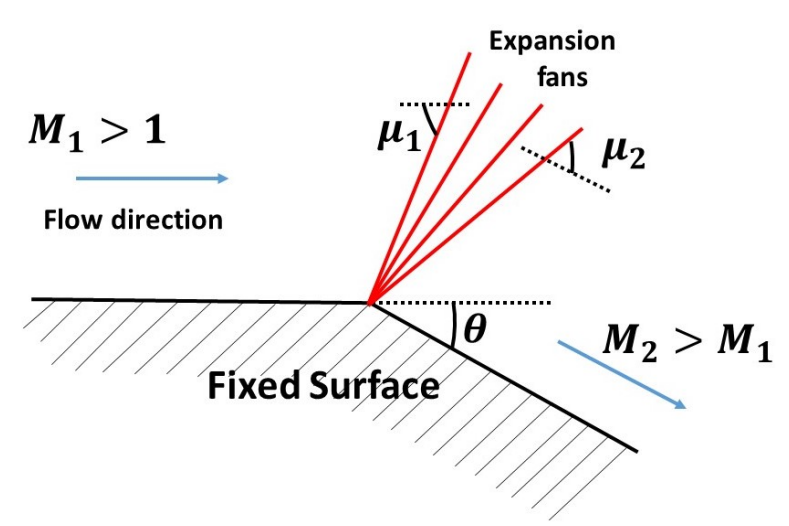

Figure 6: Schematic diagram representing expansion fan with the first and last Mach angle $\mu_{1}$ and $\mu_{2}$ respectively.

$$
\mu_{2}(M)=\sin ^{-1} \frac{1}{M_{2}}
$$

Equation (9) gives "Prandtl-Meyer function" which determines the angle through which a sonic flow must turn to reach the Mach number $M$. Equation (10) shows the relation between the Mach numbers before and after the turn; and turn angle $(\theta)$.

$$
\begin{gathered}
\nu(M)=\sqrt{\frac{\gamma+1}{\gamma-1}} \tan ^{-1} \sqrt{\frac{\gamma-1}{\gamma+1}\left(M^{2}-1\right)}-\tan ^{-1} \sqrt{M^{2}-1} \\
\theta=\nu\left(M_{2}\right)-\nu\left(M_{1}\right) .
\end{gathered}
$$

If a supersonic flow is turned abruptly and the flow area decreases resulting in an irreversible flow, the isentropic relations are no longer valid. In this case, the flow is governed by the normal or oblique shock relations as discussed in the next section. Across a shock wave; the static pressure, temperature, and gas density increases almost instantaneously. However, there is a loss of total pressure associated with a shock wave.

\subsection{Normal Shock Relations}

A normal shock occurs in front of a blunt object if the flow is supersonic and turned by a large amount, and the shock cannot remain attached to the body. Normal shock is perpendicular to the direction of flow, as shown in Figure 7. Across the normal shock, the flow changes from supersonic to subsonic conditions. Equations (11) to (17) are derived by considering the conservation of mass, momentum, and energy for a compressible fluid as given in references [10,11].

$$
\begin{gathered}
M_{2}^{2}=\frac{1+[(\gamma-1) / 2] M_{1}^{2}}{\gamma M_{1}^{2}-(\gamma-1) / 2} \\
\frac{\rho_{2}}{\rho_{1}}=\frac{U_{1}}{U_{2}}=\frac{(\gamma+1) M_{1}^{2}}{2+(\gamma-1) M_{1}^{2}}
\end{gathered}
$$




$$
\begin{gathered}
\frac{P_{2}}{P_{1}}=1+\frac{2 \gamma}{\gamma+1}\left(M_{1}^{2}-1\right) \\
\frac{T_{2}}{T_{1}}=\left(\frac{P_{2}}{P_{1}}\right)\left(\frac{\rho_{1}}{\rho_{2}}\right) \\
s_{2}-s_{1}=c_{p} \ln \frac{T_{2}}{T_{1}}-R \ln \frac{P_{2}}{P_{1}} \\
\frac{P_{02}}{P_{01}}=e^{-\left(s_{2}-s_{1}\right) / R} \\
\frac{P_{02}}{P_{1}}=\frac{P_{02}}{P_{2}} \frac{P_{2}}{P_{1}} .
\end{gathered}
$$

These equations are specialized for a one-dimensional flow assuming no heat exchange. Supersonic inlets and shock tubes are two examples where normal shocks generally occur. Figure 8 shows the user interface layout for the inputs and outputs for "normal shock relations".

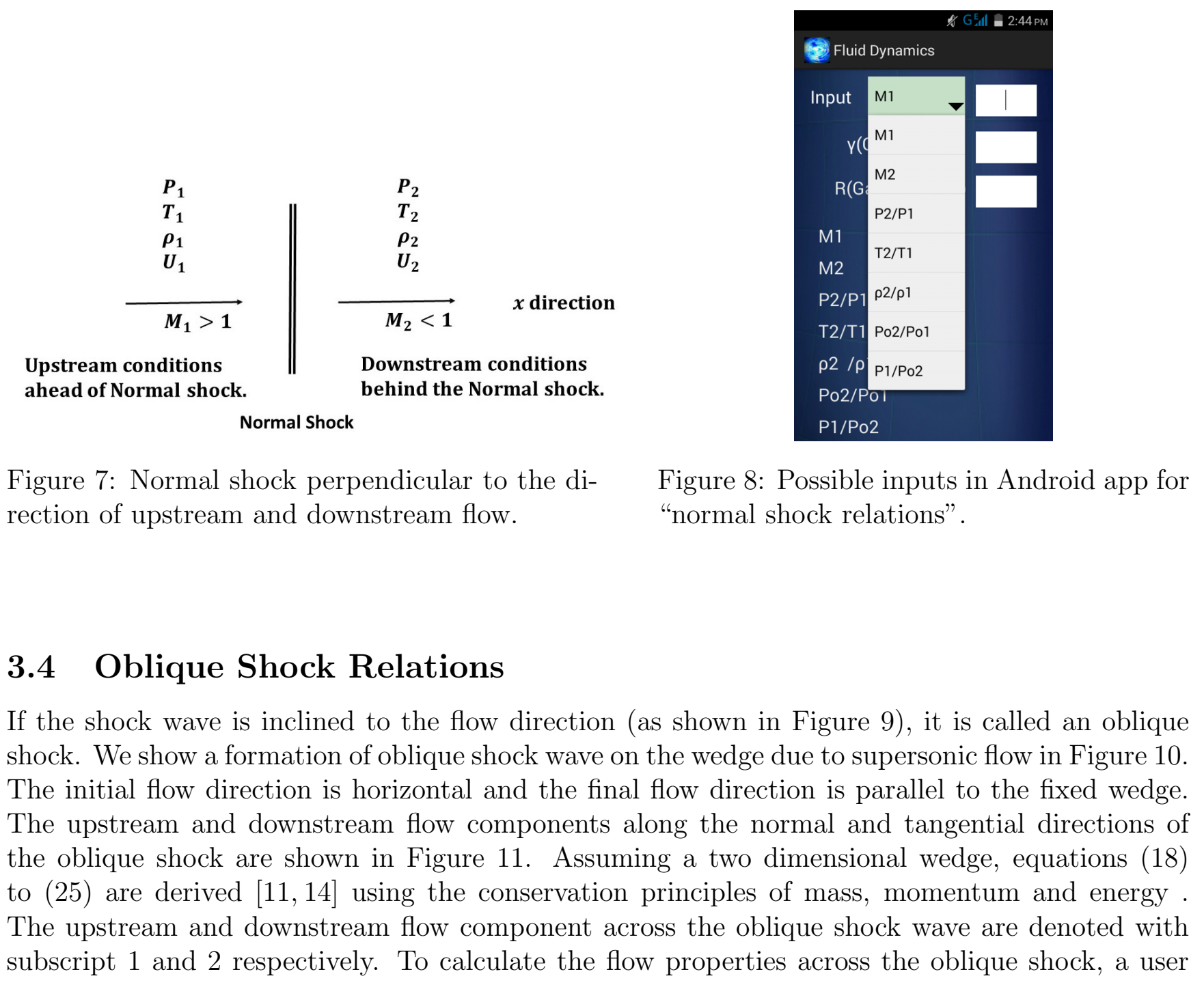




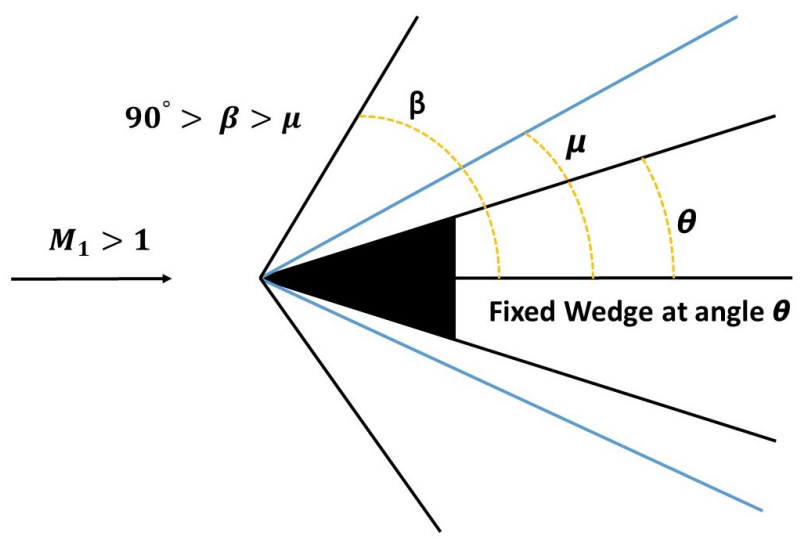

Figure 9: Supersonic flow past a two dimensional wedge generating oblique shock at an angle $\beta\left(90^{\circ}>\beta>\mu\right)$.

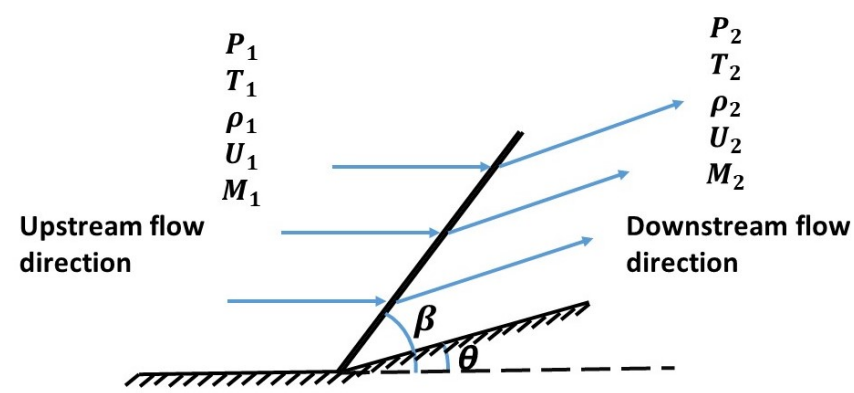

Fixed Wedge at angle $\theta$

Figure 10: Flow direction with respect to upper half surface of fixed wedge. n1 - Normal upstream component

t1 - Down stream component

n2-Normal upstream component

t2 - Down stream component

Tangential flow along

Shock wave at angle $\boldsymbol{\beta}$

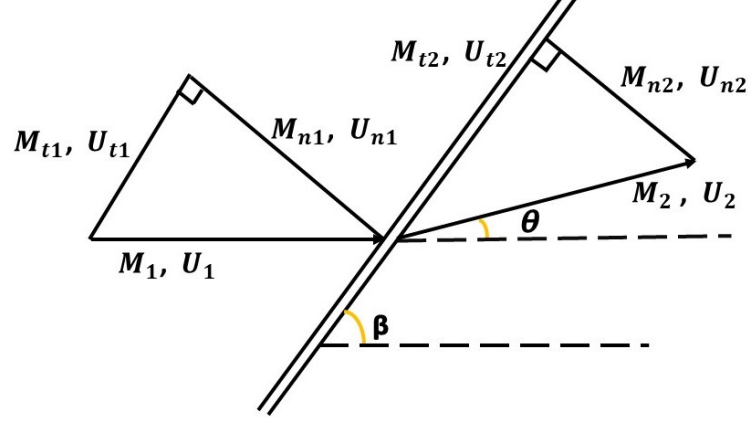

Figure 11: Normal and tangential components of inclined flow across oblique shock.

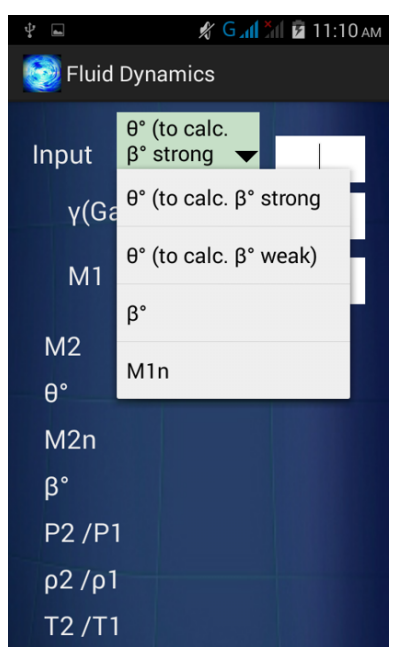

Figure 12: Possible inputs in Android app for "oblique shock relations".

interface layout of the app is shown in Figure 12. It shows the possible inputs and outputs for the flow properties across the oblique shock based on equations (18) to (25).

$$
\begin{gathered}
M_{n, 1}=M_{1} \sin \beta \\
M_{n, 2}=M_{2} \sin (\beta-\theta) \\
M_{n, 2}^{2}=\frac{1+[(\gamma-1) / 2] M_{n, 1}^{2}}{\gamma M_{n, 1}^{2}-(\gamma-1) / 2} \\
\frac{\rho_{2}}{\rho_{1}}=\frac{(\gamma+1) M_{n, 1}^{2}}{2+(\gamma-1) M_{n, 1}^{2}} \\
\frac{P_{2}}{P_{1}}=1+\frac{2 \gamma}{\gamma+1}\left(M_{n, 1}^{2}-1\right)
\end{gathered}
$$




$$
\begin{gathered}
\frac{T_{2}}{T_{1}}=\frac{P_{2}}{P_{1}} \frac{\rho_{1}}{\rho_{2}} \\
\tan \theta=2 \cot \beta \frac{M_{1}^{2} \sin ^{2} \beta-1}{M_{1}^{2}(\gamma+\cos 2 \beta)+2} \\
\frac{P_{02}}{P_{01}}=\frac{P_{02}}{P_{2}} \frac{P_{1}}{P_{01}} \frac{P_{2}}{P_{1}} .
\end{gathered}
$$

The app also calculates the shock wave-expansion fan interaction over the two dimensional diamond wedge inclined at an angle $\alpha$ (see Figure 13). The schematics of oblique shock and expansion fan over the diamond wedge are shown in Figure 14. We can see that the oblique shock waves are generated through the convergence angles $\theta_{1}$ and $\theta_{2}$ due to compression. On the other hand, the expansion fans are generated through divergence angle $\beta$ due to expansion. After passing through the expansion fans, flow further needs to pass through two more oblique shocks to align itself and to satisfy the flow properties in the back atmosphere. Given the flow upstream Mach number $M_{1}$, wedge angle $\theta$, and inclination angle $\alpha$; we calculate pressure, temperature and density ratios across the diamond wedge.

For solving this problem analytically, we use a) equations (18) to (24) for calculating the flow properties across the shocks and b) equations (1) to (10) for calculating the flow properties across the expansion fan. Computing this problem mentally using pen-paper calculations is a tedious process which is prone to errors. Further, most of the standard university textbooks have limited examples which are insufficient to validate all analytical results corresponding to a wide set of problems and their inputs. In contrast, while solving this problem through Android app for the upper half of the wedge, we just need to enter known corresponding upstream Mach number $M_{1}$ for oblique shock "a" at the leading edge. The app calculates downstream Mach number $M_{2}$ for this shock "a" which also acts as upstream Mach number for expansion fan "b". With the help of isentropic relations as discussed above, the app provides downstream Mach number $M_{3}$ for this expansion fan "b", which also acts as an upstream Mach number for oblique shock "c". Similarly, the Android app can be used to calculate Mach numbers $M_{4}$ and $M_{5}$ for the lower half of the wedge. This procedure provides us Mach numbers at all flow conditions. Further, required data such as pressure ratio, temperature ratio, density ratio, etc. can be calculated as per the user requirement from app calculation. The app solves such complex problems quickly and provides robust solutions over a wide range of given inputs.

\subsection{Fanno Flow Relations}

Fanno flow refers to an adiabatic flow through a constant area duct, where the effect of friction is considered. This has been shown in Figure 15. The flow is assumed to be steady and one dimensional, with no mass added within the duct. This flow is an irreversible process due to viscous effects. The viscous friction causes the flow properties to change along the duct. The frictional effect is modeled as a shear stress at the wall acting on the fluid with uniform properties over any cross section of the duct. As an example in Figure 16, subsonic flow is accelerated to supersonic flow through the convergent-divergent nozzle and is passed through a constant area 


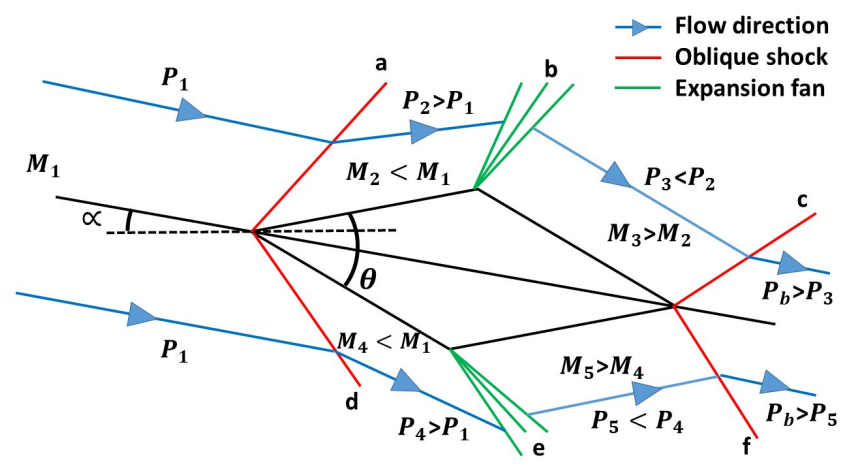

Figure 13: Two dimensional diamond wedge problem inclined at an angle $\alpha$.

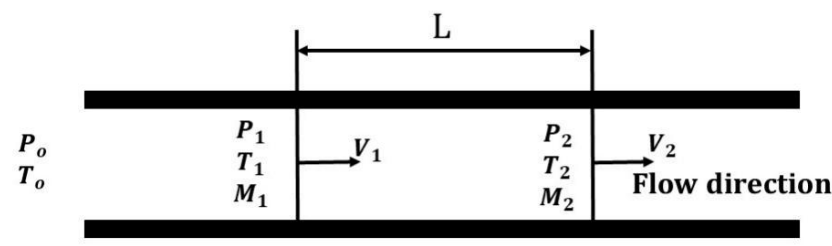

Figure 15: Adiabatic flow through a constant area duct with frictional effect.

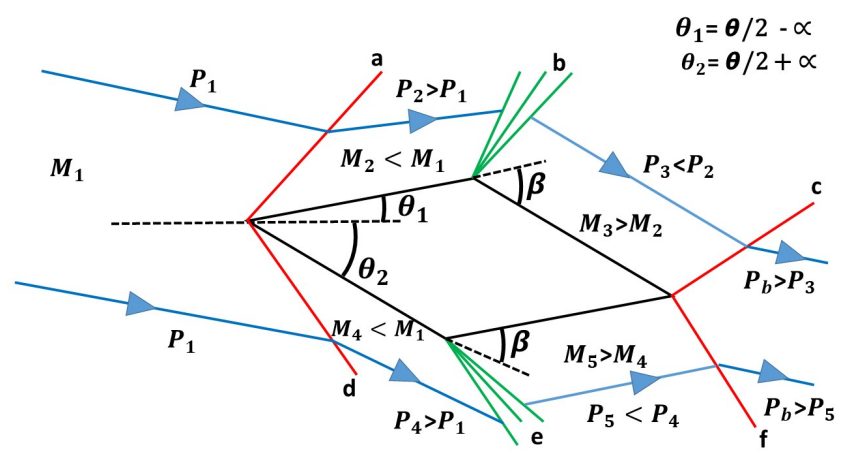

Figure 14: Flow passing through four oblique shocks and two expansions waves.

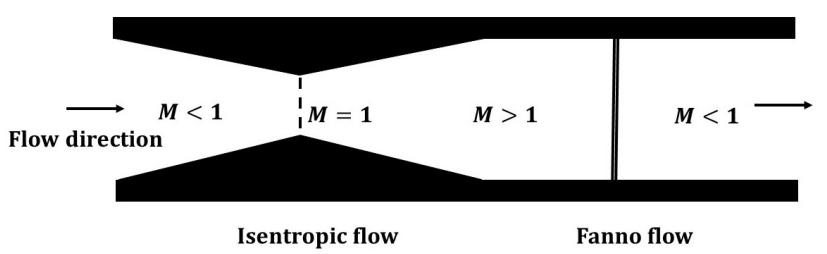

Figure 16: Supersonic flow through a constant area duct with frictional effect.

duct with a frictional effect where Fanno flow conditions are applicable. Equations (26) to (31) are derived and solved to describe Fanno flow relations with respect to the values at the choking location [10].

$$
\begin{gathered}
\frac{U}{U^{*}}=\frac{\rho^{*}}{\rho}=M(\gamma+1)^{1 / 2}\left[2\left(1+\frac{\gamma-1}{2} M^{2}\right)\right]^{-1 / 2} \\
\frac{T}{T^{*}}=(\gamma+1)\left[2\left(1+\frac{\gamma-1}{2} M^{2}\right)\right]^{-1} \\
\frac{P}{P^{*}}=\frac{1}{M}(\gamma+1)^{1 / 2}\left[2\left(1+\frac{\gamma-1}{2} M^{2}\right)\right]^{-1 / 2} \\
\frac{P_{o}}{P_{o}^{*}}=\frac{1}{M}\left[2\left(1+\frac{\gamma-1}{2} M^{2}\right)\right]^{\frac{\gamma+1}{2(\gamma-1)}}(\gamma+1)^{\frac{-(\gamma+1)}{2(\gamma-1)}} \\
\frac{s-s^{*}}{R}=-\ln \frac{1}{M}\left[\left[2\left(1+\frac{\gamma-1}{2} M^{2}\right)\right]^{\frac{\gamma+1}{2(\gamma-1)}}(\gamma+1)^{\frac{-(\gamma+1)}{2(\gamma-1)}}\right. \\
\frac{4 F L^{*}}{D}=\frac{1-M^{2}}{\gamma M^{2}}+\frac{\gamma+1}{2 \gamma} \ln \frac{(\gamma+1) M^{2}}{2\left(1+\frac{\gamma-1}{2} M^{2}\right)} .
\end{gathered}
$$


To calculate the Fanno flow relations, the user interface layout of the app is shown in Figure 17. It shows all possible inputs and outputs for the flow properties calculated using equations (26) to (31) in the Fanno flow.

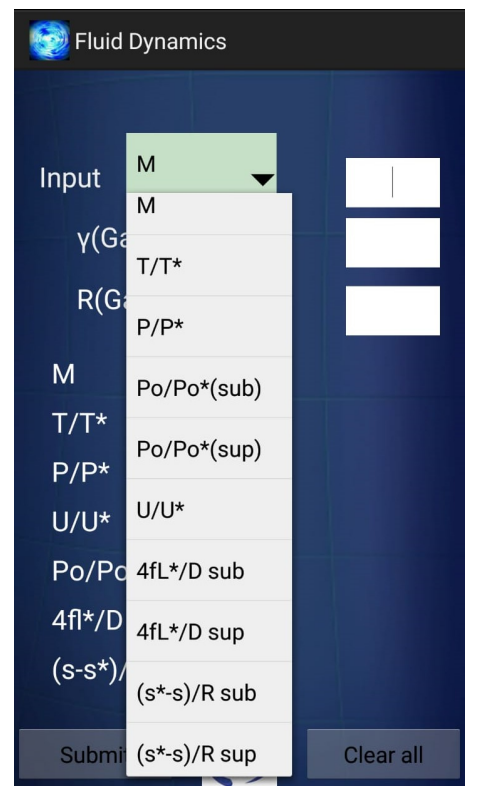

Figure 17: Possible inputs in Android app for "Fanno flow relations".

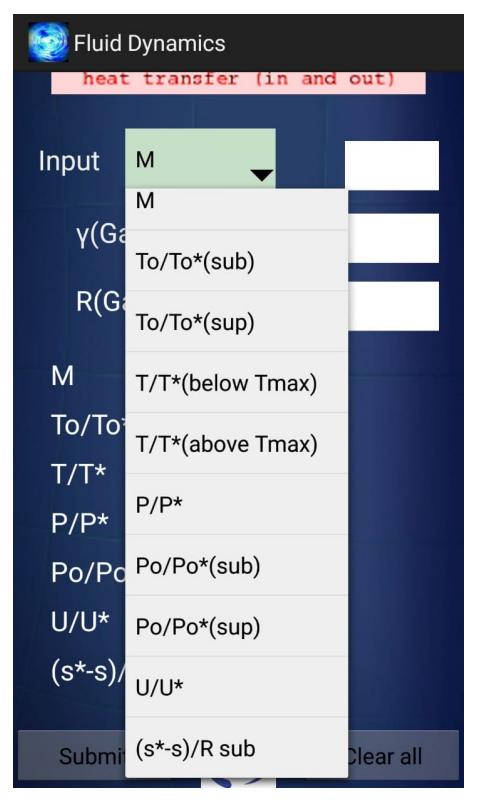

Figure 18: Possible inputs in Android app for "Rayleigh flow relations".

\subsection{Rayleigh Flow Relations}

Rayleigh Flow refers to a non-adiabatic frictionless flow through a constant area duct where the effect of heat addition or rejection is considered, and no mass is added within the duct (see Figure 19). The resulting decrease in stagnation pressure (because of heat addition) is known as the Rayleigh effect. Heat addition leads to an increase in subsonic Mach number and decrease in supersonic Mach number such that these flow approach to Mach 1. Conversely, heat rejection decreases a subsonic Mach number and increases a supersonic Mach number along the duct. In reference [10], equations (32) to (37) are derived and solved to describe Rayleigh flow property ratios with respect to the values at the choking location. To calculate the Rayleigh flow relations, the user interface layout of the app is shown in Figure 18, It shows all possible inputs and outputs for the flow properties calculated using equations (32) to (37) in the Rayleigh flow.

$$
\begin{gathered}
\frac{P}{P^{*}}=\frac{1+\gamma}{1+\gamma M^{2}} \\
\frac{P_{o}}{P_{o}^{*}}=\frac{1+\gamma}{1+\gamma M^{2}}\left[2\left(1+\frac{\gamma-1}{2} M^{2}\right)\right]^{\frac{\gamma}{\gamma-1}}(\gamma+1)^{\frac{-\gamma}{\gamma-1}} \\
\frac{T}{T^{*}}=M^{2}\left(\frac{P}{P^{*}}\right)^{2}
\end{gathered}
$$




$$
\begin{gathered}
\frac{T_{o}}{T_{o}^{*}}=2\left(\frac{P}{P^{*}}\right) \frac{M^{2}}{1+\gamma M^{2}}\left(1+\frac{\gamma-1}{2} M^{2}\right) \\
\frac{U^{*}}{U}=\frac{\rho}{\rho^{*}}=\frac{1+\gamma M^{2}}{(1+\gamma) M^{2}} \\
\frac{s^{*}-s}{R}=\ln \left[\left(\frac{1}{M}\right)^{2 \gamma /(\gamma-1)}\left(\frac{1+\gamma M^{2}}{1+\gamma}\right)^{\frac{\gamma+1}{\gamma-1}}\right] .
\end{gathered}
$$

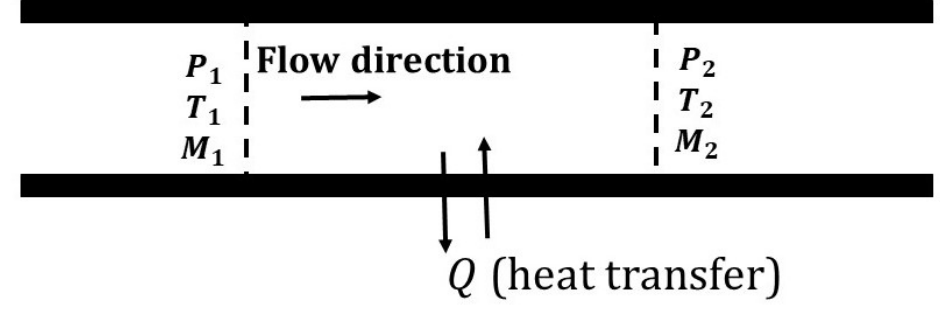

Figure 19: Non-Adiabatic frictionless flow through a constant area duct with heat transfer.

\subsection{International Standard Atmosphere (ISA) Relations}

The earth's atmosphere is a dynamically changing system. To model it, U.S. Air Force provided standard atmosphere relations in 1959. International Standard Atmosphere (ISA) is a static atmospheric model of how the pressure $P$, temperature $T$, and density $\rho$ of the Earth's atmosphere change over a wide range of altitudes. The model comprises a number of standard definitions for the altitudes. The geometric altitude $H_{G}$ is the standard height above the mean sea level. The geopotential altitude $H_{G P}$ is calculated from a mathematical model that adjusts the altitude to include the variation of gravity with height. Figure 20 shows temperature distribution with respect to geopotential altitude. The relations applicable in various regimes of this atmospheric model are given by equations 38 to 45 .

$$
\begin{gathered}
H_{G P}=\left(\frac{R}{R+H_{G}}\right) H_{G} \\
g=g_{o}\left(\frac{R}{R+H_{G}}\right)^{2} .
\end{gathered}
$$

For gradient layers,

$$
\begin{gathered}
\frac{T-T_{b}}{H_{G P}-H_{b}}=\frac{d T}{d H_{G P}}=a \\
\frac{P}{P_{b}}=\left(\frac{T}{T_{b}}\right)^{-g_{o} / a R}
\end{gathered}
$$




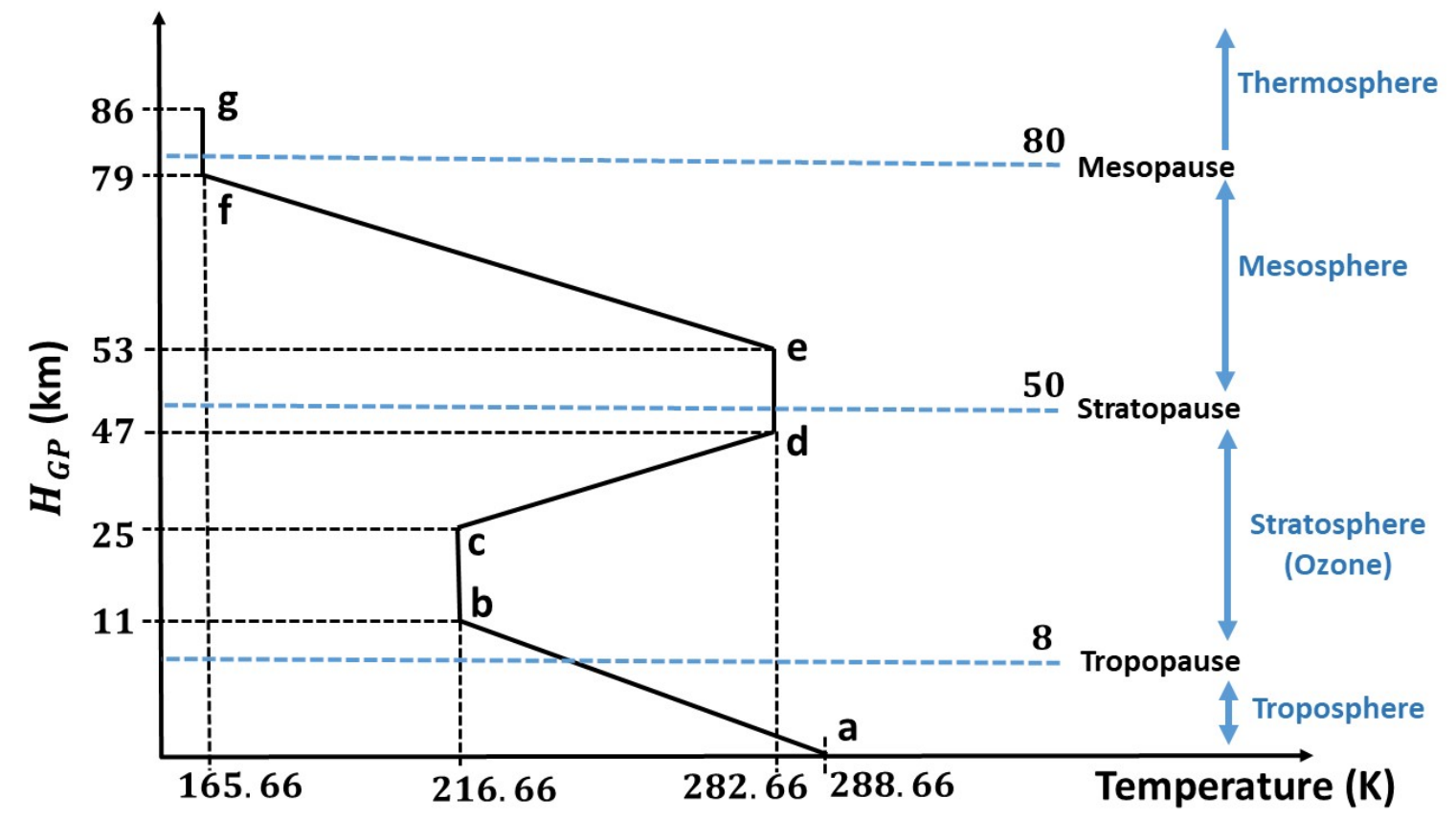

Figure 20: Temperature distribution in standard atmosphere.

$$
\begin{aligned}
& \frac{\rho}{\rho_{b}}=\left(\frac{T}{T_{b}}\right)^{-\left[\left(g_{o} / a R\right)+1\right]} \\
& T=T_{b}+a\left(H_{G P}-H_{b}\right) .
\end{aligned}
$$

For isothermal layers,

$$
\begin{aligned}
& \frac{P}{P_{b}}=e^{\left(-g_{o} / R T\right)\left(H_{G P}-H_{b}\right)} \\
& \frac{\rho}{\rho_{b}}=e^{\left(-g_{o} / R T\right)\left(H_{G P}-H_{b}\right)} .
\end{aligned}
$$

Table 1 corresponds to standard lapse rate and base values of temperature, pressure and density at different atmospheric regimes.

\begin{tabular}{|c|c|c|c|c|c|}
\hline Range & $H_{G P}($ in $\mathrm{km})$ & a (in $\mathrm{K} / \mathrm{km})$ & $T_{B}$ (in Kelvin) & $P_{B}($ in Pascals) & $\rho_{B}\left(\mathrm{in} \mathrm{kg} / \mathrm{m}^{3}\right)$ \\
\hline$a-b$ & $<11$ & -6.5 & 288.16 & $1.013 \times 10^{5}$ & 1.225 \\
\hline$b-c$ & $11-25$ & 0 & 216.66 & 22700 & 0.3648 \\
\hline$c-d$ & $25-47$ & 3 & 216.66 & 2527.3 & 0.0406 \\
\hline$d-e$ & $47-53$ & 0 & 282.66 & 125.58 & 0.0015 \\
\hline$e-f$ & $53-79$ & -4.5 & 282.66 & 61.493 & 0.000758 \\
\hline$f-g$ & $79-86$ & 0 & 165.66 & 1.2437 & 0.000022 \\
\hline
\end{tabular}

Table 1: Standard lapse rate and base value of temperature, pressure and density for different ranges in atmosphere. 
To calculate the ISA relations, the user interface layout of the app is shown in Figure 21. It shows all possible inputs and outputs for the flow properties calculated using equations (38) to 45) in the standard atmospheric model.

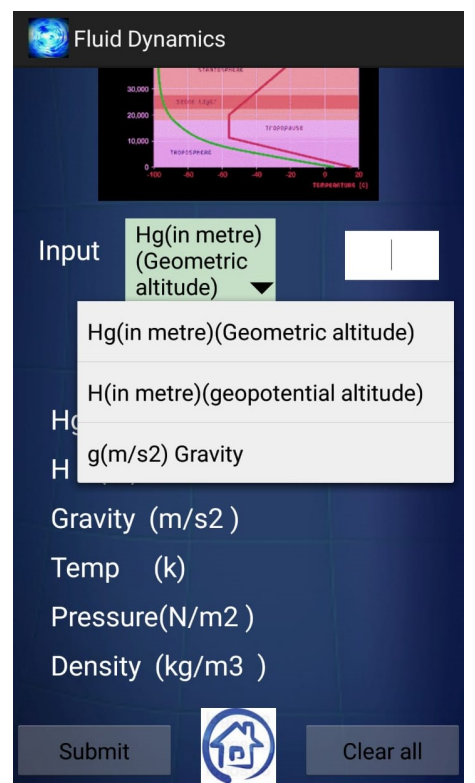

Figure 21: Possible inputs in Android app for "International Standard Atmosphere (ISA) relations".

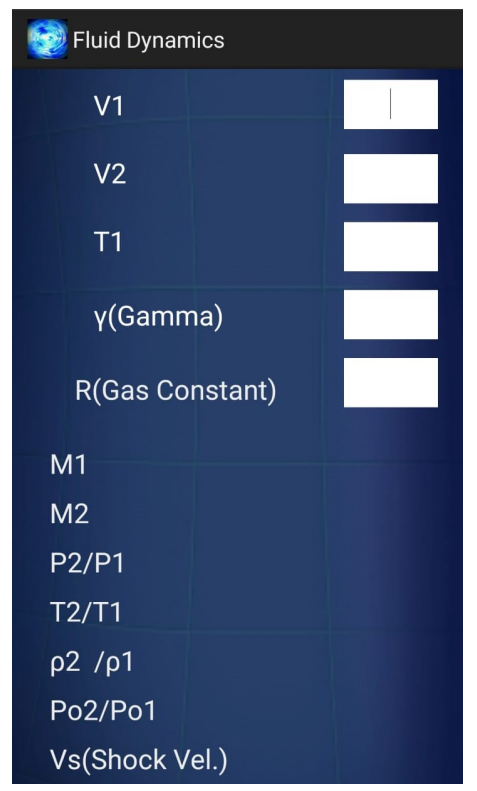

Figure 22: Possible inputs in Android app for "moving normal shock relations".

\subsection{Moving Normal Shock Relations}

The app is able to calculate flow across moving normal shock waves which are generated in the shock-tube or by an explosion. We show the schematic diagram of moving normal shock in stationary frame of reference in Figure 23. In this figure, the shock is moving with the velocity $V_{s}$. $V_{1}$ and $V_{2}$ represent the upstream and the downstream flow velocity across moving normal shock respectively. For the moving normal shock, we assume $U_{1}=V_{s}-V_{1}$ and $U_{2}=V_{s}-V_{2}$. Substituting these values in equation 12 , we get

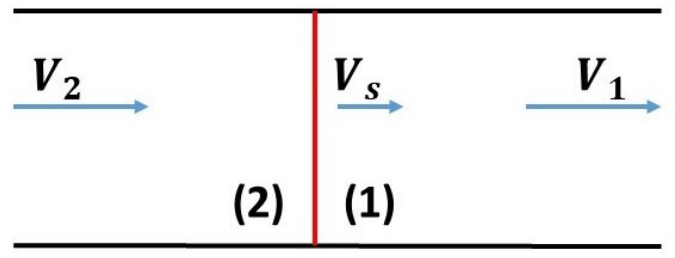

Figure 23: Moving normal shock (red) in stationary coordinate system.

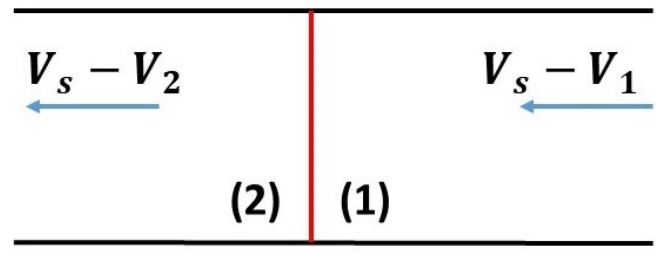

Figure 24: Moving normal shock (red) in moving coordinate system.

$$
\frac{\rho_{2}}{\rho_{1}}=\frac{V_{s}-V_{1}}{V_{s}-V_{2}}=\frac{(\gamma+1) M_{1}^{2}}{2+(\gamma-1) M_{1}^{2}}
$$


Both $V_{1}$ and $V_{2}$ are known parameters. $V_{s}$ is the unknown shock velocity. We show the schematic diagram of moving normal shock in moving frame of reference with the shock in Figure 24. Given the upstream Mach number $M_{1}$, temperature $T_{1}$, and velocities $V_{1}$ and $V_{2}$, we calculate shock speed $V_{s}$ from equation (46). Further, Equations (13), (14) and (46) are used to calculate pressure, temperature and density ratios across moving normal shock respectively. To calculate the flow properties across moving shock normal shock wave, the user interface layout for all possible inputs and outputs of the app is shown in Figure 22.

\subsection{Pitot Tube Relations}

A Pitot tube is a fluid flow measuring device, used to measure the airspeed of an aircraft. It consists of a tube pointing directly to the fluid flow. The moving fluid comes to a rest since there is no outlet for a continuous flow. The pressure corresponding to the stationary flow is called stagnation pressure of the fluid, also known as the Pitot pressure of the tube. The pressure corresponding to the flowing flow is called the static pressure of the flow. If the aircraft is flying at subsonic speed, we use isentropic flow condition across the Pitot tube. On the other hand, if the aircraft flies with a supersonic speed, then a normal shock stands in front of the tip of the Pitot tube. In this case, the procedure to calculate the airspeed becomes different giving rise to Rayleigh Pitot tube formulation. Both the formulations are described as follows. For subsonic flow, we use isentropic relations (see Figure 25), i.e., equation (3), and get

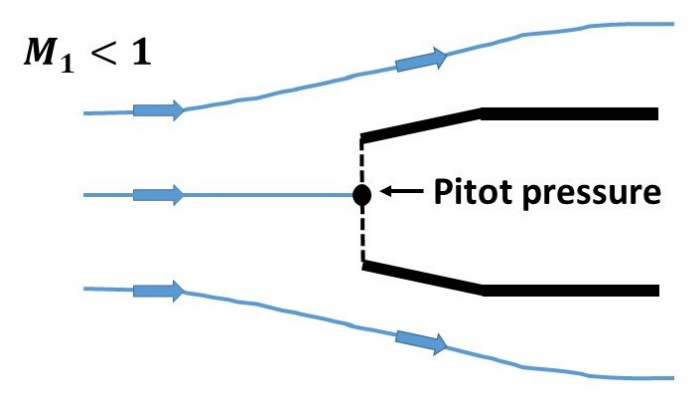

Figure 25: Subsonic flow : Pitot pressure is the free stream total pressure $P_{o, 1}$.

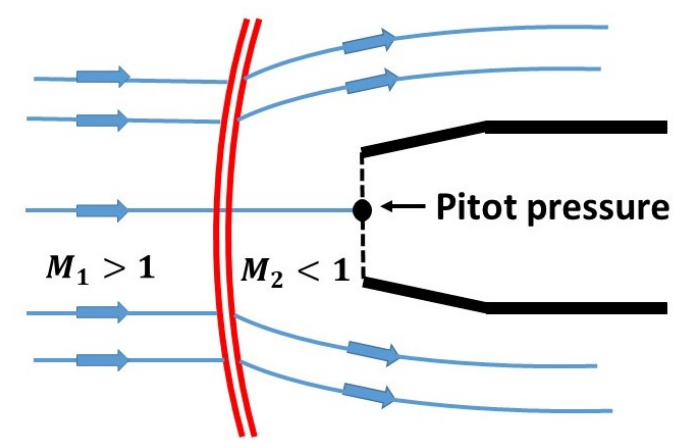

Figure 26: Supersonic flow : Pitot pressure is the total pressure behind a normal shock $P_{o, 2}$.

$$
\begin{gathered}
\frac{P_{01}}{P_{1}}=\left(1+\frac{\gamma-1}{2} M_{1}^{2}\right)^{\frac{\gamma}{\gamma-1}} \Longrightarrow M_{1}^{2}=\frac{2}{\gamma-1}\left[\left(\frac{P_{o, 1}}{P_{1}}\right)^{\frac{\gamma-1}{\gamma}}-1\right] \\
U_{1}^{2}=\frac{2 a_{1}^{2}}{\gamma-1}\left[\left(\frac{P_{o, 1}}{P_{1}}\right)^{\frac{\gamma-1}{\gamma}}-1\right] .
\end{gathered}
$$

For supersonic flow, where a normal shock occurs in front of the tip as shown in Figure 26, the Pitot tube measures the total pressure downstream of the shock and the downstream static pressure. Therefore, in order to get the upstream Mach number, we re-write the Pitot tube measurements in terms of the upstream flow variables as

$$
\frac{P_{o, 2}}{P_{1}}=\frac{P_{o, 2}}{P_{2}} \frac{P_{2}}{P_{1}} .
$$


Substituting $P_{o, 2} / P_{2}$ and $P_{2} / P_{1}$ from equation (3) and (13) respectively, we get

$$
\frac{P_{o, 2}}{P_{1}}=\left(\frac{(\gamma+1)^{2} M_{1}^{2}}{4 \gamma M_{1}^{2}-2(\gamma-1)}\right)^{\gamma / \gamma-1} \frac{1-\gamma+2 \gamma M_{1}^{2}}{\gamma+1}
$$

This is known as Rayleigh-Pitot tube formula. To calculate the flow properties across Pitot tube in both compressible (with or without shock) flow and incompressible flow, the user interface layout for all possible inputs and outputs of the app is shown in Figure 27(a,b,c).

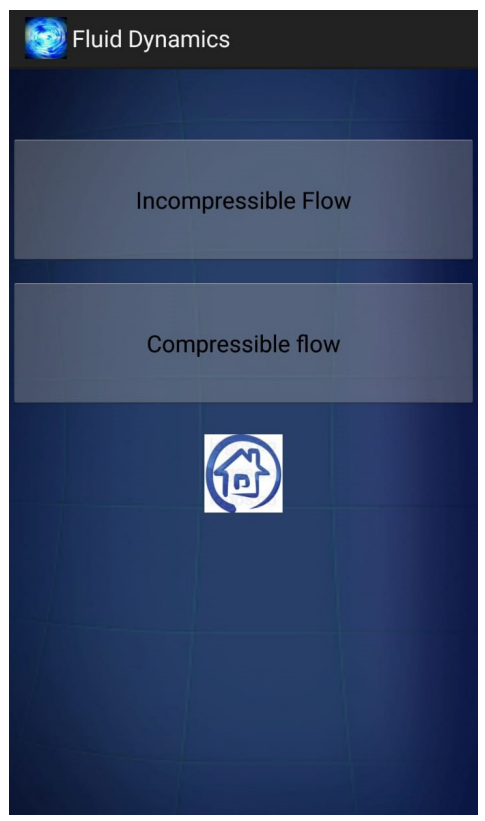

(a) Snapshot after the selection of "Pitot tube" relations.

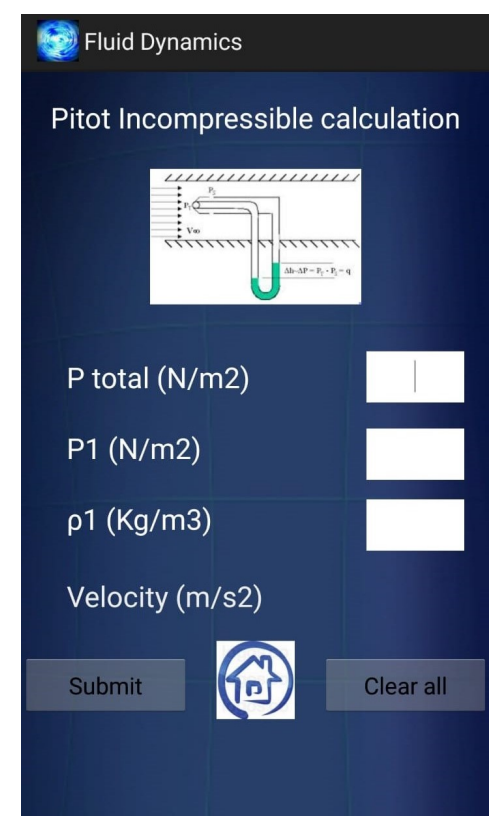

(b) Snapshot after the selection of "incompressible flow".

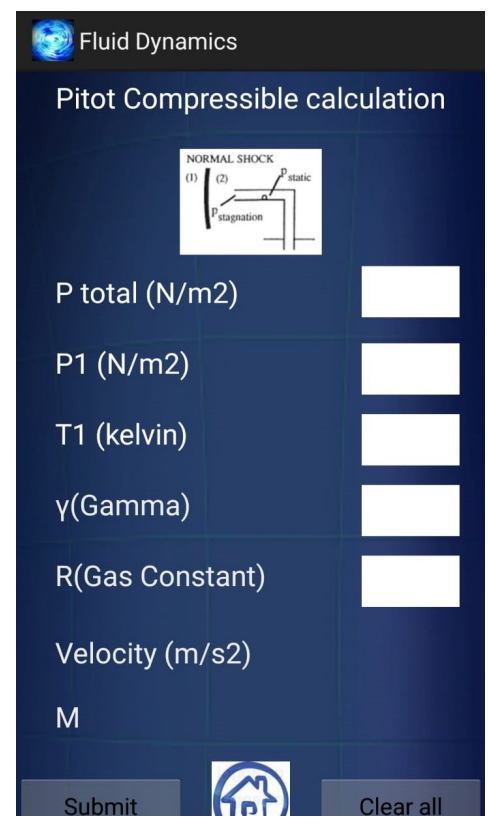

(c) Snapshot after the selection of "compressible flow".

Figure 27: Series of snapshots depicting complete representation for Pitot tube formulations for both compressible flow and incompressible flow.

\section{Implementation of Numerical Methods}

In this section, we discuss the numerical methods which we have implemented in this app to solve the semi-implicit formulations presented in the previous section. As an example we show the procedure to calculate the solution of equation (5) using Newton-Raphson method and equation (24) using Bisection method respectively within the app. These methods are further extended to calculate the solution for other implicit formulations in the "Fluid Dynamics" Android application.

\subsection{Newton-Raphson Method}

Given the inputs, critical area ratio $A / A^{*}=5.5$ and specific heat constant ratio $\gamma=1.4$, the app algorithm calculates and returns corresponding subsonic and supersonic Mach number results using the Newton-Raphson method in equation (5). The procedure follows: As we know the equation 
(5) is

$$
\left(\frac{A}{A^{*}}\right)^{2}=\frac{1}{M^{2}}\left[\frac{2}{\gamma+1}\left(1+\frac{\gamma-1}{2} M^{2}\right)\right]^{\frac{(\gamma+1)}{(\gamma-1)}}
$$

The above equation can be re-written as

$$
f(M)=\left[\frac{2}{\gamma+1}\left(1+\frac{\gamma-1}{2} M^{2}\right)\right]^{\frac{(\gamma+1)}{2(\gamma-1)}}-M\left(\frac{A}{A^{*}}\right)=0 .
$$

For finding the roots of the function $f(M)$ of equation (51), the algorithm starts with an initial guess $M_{\text {old }}$ and applies Newton-Raphson approach as

$$
M_{\text {new }}=M_{\text {old }}-\frac{f\left(M_{\text {old }}\right)}{\frac{d f}{d M}\left(M_{\text {old }}\right)} .
$$

For solving above equation (52), the algorithm calculates derivative of equation (51) as

$$
\frac{d f}{d M}(M)=\frac{A}{A^{*}}-\left(\frac{2}{\gamma+1}\right)^{\frac{\gamma+1}{2(\gamma-1)}}(\gamma-1) M\left(1+\frac{\gamma-1}{2} M^{2}\right)^{\frac{(\gamma+1)}{2(\gamma-1)}-1} .
$$

Using the initial guess $M_{\text {old }}$ in equations (52) and (53), we get $M_{\text {new }}$ in the first step. As the procedure is having an iterative algorithm, we assign $M_{n e w}$ to $M_{\text {old }}$ in the next step and repeat the above procedure which gives a better approximation for $M_{\text {new }}$. The same process is repeated til convergence, i.e., til $\left|M_{\text {new }}-M_{\text {old }}\right| \leq 10^{-6}$.

For finding the supersonic solution for equation (51), the initial guess is taken as $M=5$, since this is the maximum possible supersonic Mach number. For finding the subsonic solution for equation (51), the initial guess is taken as $M=0.999$, since this is the possible critical Mach number for subsonic solution. The selection of $\left(A / A^{*}\right)_{\text {sup }}$ and $\left(A / A^{*}\right)_{\text {sub }}$ in Figure $3 \mathrm{a}$ leads to supersonic and subsonic solutions respectively.

For any given problem, where $A / A^{*}=5.5$ and $\gamma=1.4$ are known inputs, $M=3.2755$ and $M=0.1059$ are displayed as supersonic and subsonic solutions respectively.

\subsection{Bisection Method}

Equation (24) represents the relation between $\theta, \beta$ and $M$. It is solved using the Bisection method which gives two solutions for solving wave (shock) angle. The higher and the lower values correspond to the strong and the weak shock solutions respectively. Therefore, two different inputs, " $\theta$ (to calculate $\beta$ strong)" and " $\theta$ (to calculate $\beta$ weak)" are provided as shown in Figure 12 , Depending on the input selected by the user, corresponding outputs are displayed.

Given the inputs; upstream Mach number $M_{1}$, specific heat constant ratio $\gamma$ and deflection angle $\theta$, app algorithm outputs wave(shock) angle $\beta$. Considering equation (24),

$$
\begin{gathered}
\tan \theta=2 \cot \beta \frac{M_{1}^{2} \sin ^{2} \beta-1}{M_{1}^{2}(\gamma+\cos 2 \beta)+2} \\
f(\beta)=\tan \theta-2 \cot \beta \frac{M_{1}^{2} \sin ^{2} \beta-1}{M_{1}^{2}(\gamma+\cos 2 \beta)+2}=0
\end{gathered}
$$


if the user selects " $\theta$ (to calculate $\beta$ strong)", i.e., solving strong shock solution, then the algorithm starts with two guesses for the shock angle $\beta_{1}$ and $\beta_{2}$ such that $f\left(\beta_{1}\right) \times f\left(\beta_{2}\right)<0$. The initial guess for both $\beta_{1}$ and $\beta_{2}$ is Mach angle $\mu$ (i.e. equation (8)) and $90^{\circ}$ respectively.

For all of the above input cases, the range of the solution is $\left[\beta_{1}, \beta_{2}\right]$. As the Bisection method is an iterative method, therefore the range is reduced iteratively till $f\left(\frac{\beta_{1}+\beta_{2}}{2}\right) \approx 0$. In bisection method, the next iteration starts with the mid point of $\beta_{1}$ and $\beta_{2}$ as

$$
\beta_{s}=\frac{\beta_{1}+\beta_{2}}{2}
$$

If $f\left(\beta_{s}\right)$ is sufficiently small as per tolerance $10^{-6}$, i.e. $\left|f\left(\beta_{s}\right)\right| \leq 10^{-6}$ then it is returned as the solution. If not, then we check whether

$$
\operatorname{sign}\left(f\left(\beta_{s}\right)\right)==\operatorname{sign}\left(f\left(\beta_{1}\right)\right) .
$$

If the above condition is true then we replace $\beta_{1}$ with $\beta_{s}$ otherwise $\beta_{2}$ is replaced with $\beta_{s}$. The next iteration continues by taking mid point of the new range. The iterations continue till the required tolerance of $10^{-6}$ is achieved.

The number of iterations $(N)$ required to converge to the root within this tolerance is given as

$$
N=\frac{\ln \left(\frac{\beta_{2}-\beta_{1}}{10^{-6}}\right)}{\ln 2}
$$

\section{Results and Comparisons}

In this section, we compare the results of the Android app with the data in references [14 16. The results from the Android app and references are plotted as "App. Data" and "Ref. data" respectively. However, as stated earlier, $\gamma$ varies from 1 to 1.67, and local gas constant $R$ can also be chosen as per flow problem which ensures a wide range of variables for the app. Therefore, for the validation purpose, we use $\gamma=1.4$ and $R=287$ and compare the results against the literature. As discussed in Section 2, equations (1) to (37) are used to make these comparisons where Mach number is taken as input, and the corresponding outputs are plotted.

\subsection{Isentropic Flows and Prandtl - Meyer Expansion Fan}

In Figure 28, Mach number is plotted against $T_{o} / T, P_{o} / P$ and $\rho_{o} / \rho$ using equations (2) to (4). Similarly, in Figure 29, Mach number is plotted against $A / A^{*}$ using equation (5). We find that the results obtained from the app are in very good agreement with the results given in the literature 14 16. This validates that the implementations of the isentropic flows relations and the Prandtl Meyer expansion fan relations in the app are correct. Also these results from the app are obtained in a fraction of second which shows that the algorithms written in the app are highly efficient.

\subsection{Normal Shock Properties}

In Figure 30, upstream Mach number $M_{1}$ is plotted against downstream Mach number $M_{2}$, and temperature ratio $T_{2} / T_{1}$ using equations (11) and (14) respectively. Similarly, in Figure 31, upstream Mach number $M_{1}$ is plotted against density ratio $\rho_{2} / \rho_{1}$, static pressure ratio $P_{2} / \bar{P}_{1}$, and 


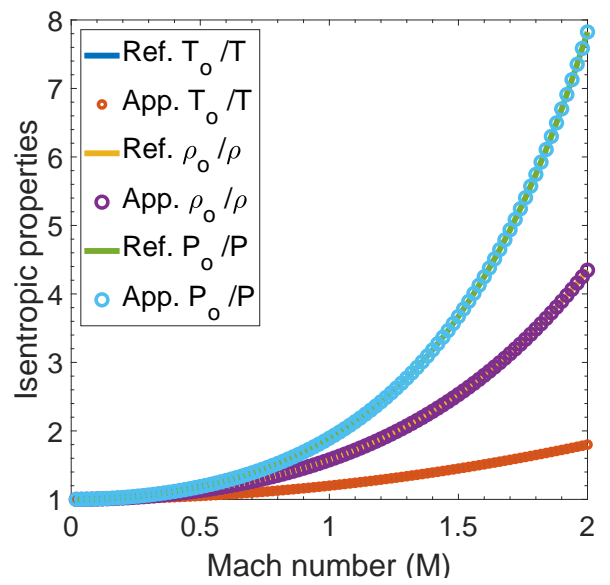

Figure 28: Isentropic relations for Mach number ranging from subsonic to supersonic values.

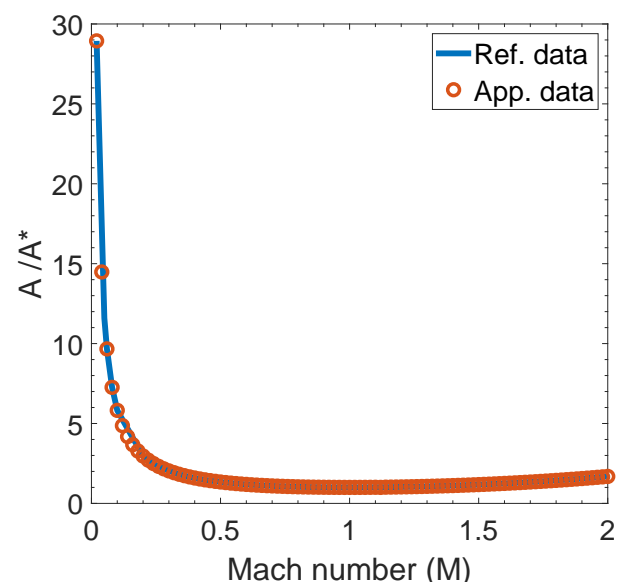

Figure 29: Geometric area to throat area ratio when flow is choked at throat for convergent-divergent nozzle.

total pressure ratio $P_{o 2} / P_{o 1}$ using equations (12), (13) and (16) respectively. We again find that the results obtained from the app are in very good agreement with the results given in the literature $[14-16]$ which validates that the implementations of the normal shock relations in the app are correct. Also these results from the app are obtained in a fraction of second which shows that the algorithms written for the normal shock relations in the app are highly efficient.

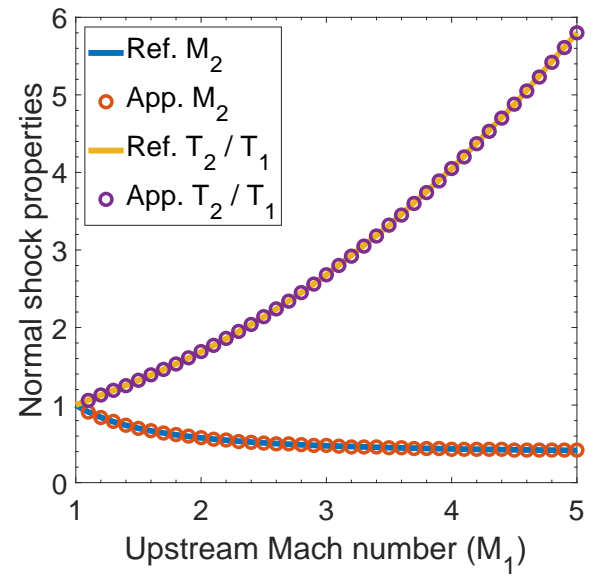

Figure 30: Relation between upstream and downstream Mach numbers and temperature ratio for normal shock.

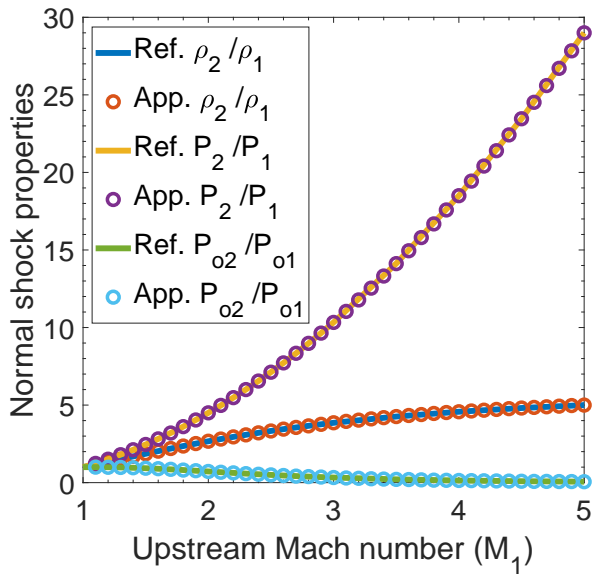

Figure 31: Relation between upstream and downstream flow properties of pressure and density ratio for normal shock.

\subsection{Oblique Shock Relations}

In Figure 32, shock wave angle $\beta$ is plotted against deflection angle $\theta$ for upstream Mach number $M=2$ and $M=5$ using equation (24). Here, minimum value of the wave angle $\beta$ is calculated using Mach angle relation $\mu=\sin ^{-1}(1 / M)$. We find that the results obtained from the app are in very good agreement with the results given in the literature 14 which validates that the 
implementations of the oblique shock relations in the app are correct. Also these results from the app are obtained in a fraction of second which shows that the algorithms written for the oblique shock relations in the app are highly efficient.

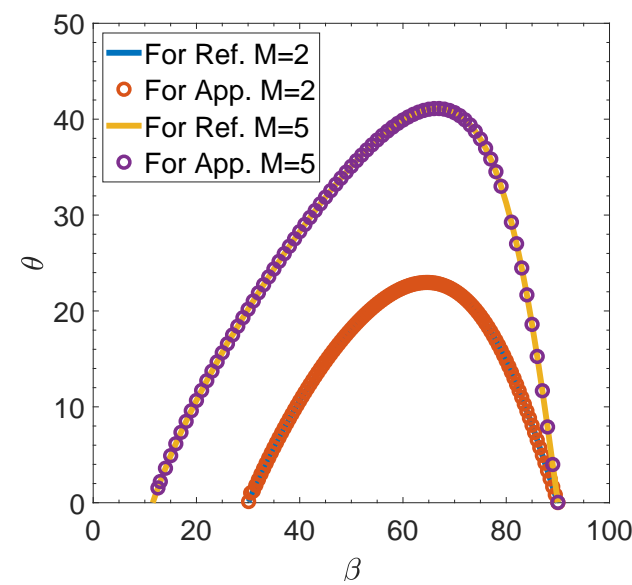

Figure 32: $\theta, \beta$ and Mach relation for oblique shock.

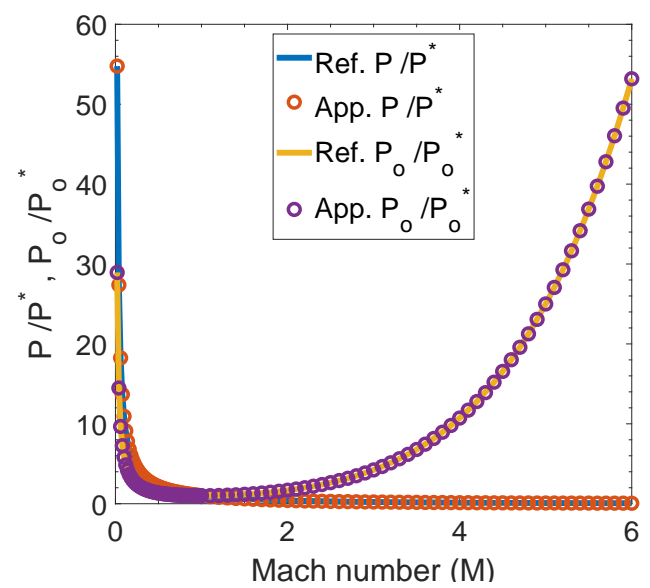

Figure 33: Static pressure ratios, total pressure ratios and Mach number at sonic condition for Fanno flow.

\subsection{Fanno Flow Relations}

In Figure 33, Mach number $M$ is plotted against static pressure ratio $P / P^{*}$ and stagnation pressure ratio $P_{o} / P_{o}{ }^{*}$ at sonic condition using equations (28) and (29) respectively. In Figure 34, Mach number $M$ is plotted against velocity ratio $U / U^{*}$ and temperature ratio $T / T^{*}$ at sonic condition using equations (26) and (27) respectively. Similarly, in Figure 35, Mach number $M$ is plotted against Fanno parameter $4 F L^{*} / D$ using equation (31). We find that the results obtained from

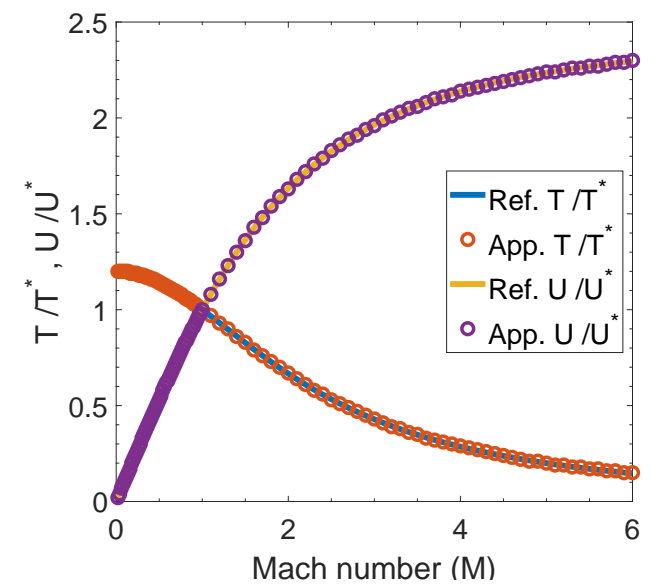

Figure 34: Static temperature ratios, velocity ratios and Mach number at sonic condition for Fanno flow.

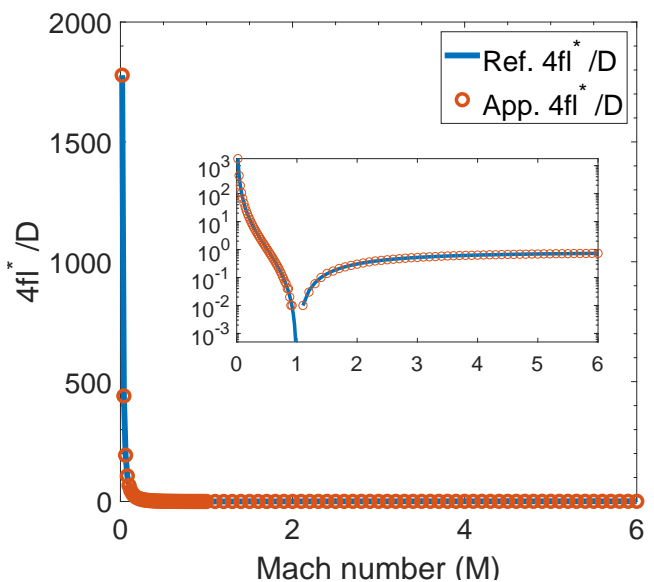

Figure 35: Fanno parameter and Mach number at sonic condition for Fanno flow.

the app are in very good agreement with the results given in the literature $[14-16]$ which validates 
that the implementations of the Fanno flow relations in the app are correct. Also these results from the app are obtained in a fraction of second which shows that the algorithms written for the Fanno flow relations in the app are highly efficient.

\subsection{Rayleigh Flow Relations}

In Figure 36, Mach number $M$ is plotted against static pressure ratio $P / P^{*}$, static temperature ratio $T / T^{*}$ and stagnation temperature ratio $T_{o} / T_{o}^{*}$ at sonic condition using equation (32), (34) and (35) respectively. In Figure 37, Mach number $M$ is plotted against stagnation pressure ratio $P_{o} / P_{o}^{*}$ and velocity ratio $U / U^{*}$ at sonic condition using equation (33) and (36) respectively.

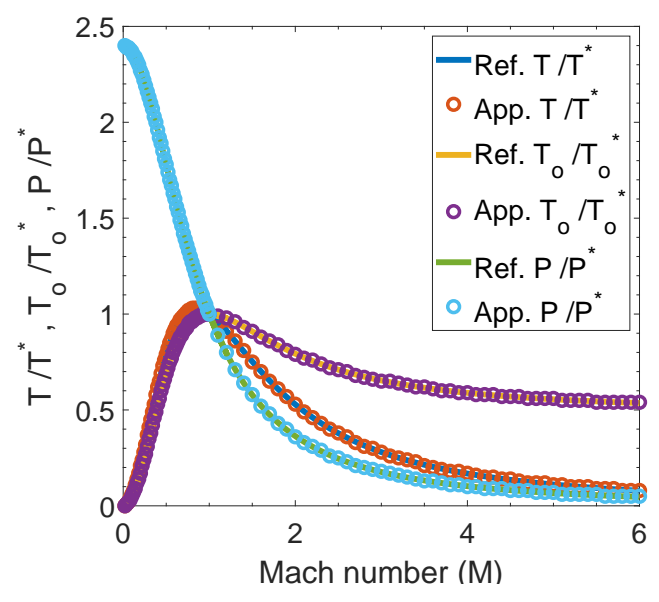

Figure 36: Correlation between static temperature ratios, total temperature ratios, static pressure ratios with Mach number at sonic condition for Rayleigh flow.

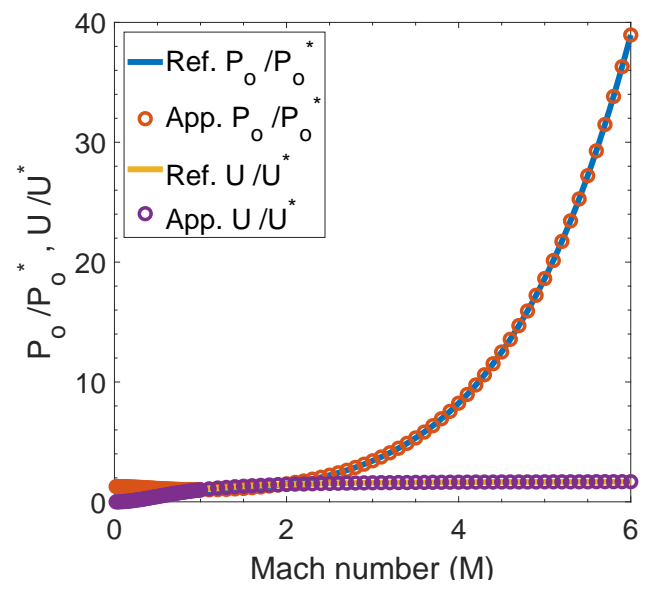

Figure 37: Correlation between total pressure ratios, velocity ratios with Mach number at sonic condition for Rayleigh flow

We find that the results obtained from the app are in very good agreement with the results given in the literature $[14-16]$ which validates that the implementations of the Rayleigh flow relations in the app are correct. Also these results from the app are obtained in a fraction of second which shows that the algorithms written for the Rayleigh flow relations in the app are highly efficient.

As shown above in all cases, the comparison between App data and Ref data (Figures 28 to 37 shows that "Fluid Dynamics" is accurate with an absolute error of $10^{-6}$; when Mach number, $\gamma=1.4$ and local gas constant $R=287$ are taken as input. However, any possible combination of inputs produces results with similar accuracy in a fraction of seconds.

\section{Conclusion}

We have designed an Android app "Fluid Dynamics", for conveniently solving the gas dynamics formulations. The app has been proven to be highly accurate in measuring the fluid properties of a variety of flows, as shown in above discussed results. Amongst all the formulations discussed above, calculations of normal and oblique shocks are the most complex ones. Such calculations are repetitive, lengthy, and time-consuming; hence cumbersome for students, scholars, and educators working in this area. Our app solves these complex formulations very fast (in a fraction of second) 
with high accuracy. The user just needs to enter inputs, and all the flow properties are calculated on one click. Future work includes extending the "Fluid Dynamics" to potential flows.

In addition to solving complex formulations, the significance of this app is also to motivate educators to develop complex domain-specific apps. The increased computational power of smartphones can be unleashed in the education system with the help of such apps. While the first-class organizations like NASA have set examples of utilizing the power of these seemingly tiny metal boxes in space, their power has not reached full potential in the mainstream education system and demands educators and researchers employ their expertise in this direction.

\section{Author Contributions}

A.G. conceived the idea of designing such a highly specific application for solving compressible flow formulation on the Android platform. S.S. and A.G. contributed to all phases of the project, from the design of the architectural framework of the Android application, to the implementation of the numerical methods and validation of the application results with benchmark. S.S., Y.G., and A.G. contributed significantly to the preparation of the submitted manuscript preceded by a number of brainstorming sessions.

\section{Conflicts of Interest}

There are no conflicts to declare.

\section{Acknowledgements}

Many thanks to 'GATE Aerospace Forum Educational Services' for their financial support. We also thank Komal Mehta for proof reading some part of the validation work.

\section{References}

[1] Mr Jared Rayleigh Wilson and Kurt C Gramoll. Viscous fluid dynamics app for mobile devices using a remote high per-formance cluster. age, 26:1, 2015.

[2] Kristine Dery, Darl Kolb, and Judith MacCormick. Working with connective flow: how smartphone use is evolving in practice. European Journal of Information Systems, 23(5):558$570,2014$.

[3] Vlad Oncescu, Dakota O'Dell, and David Erickson. Smartphone based health accessory for colorimetric detection of biomarkers in sweat and saliva. Lab on a Chip, 13(16):3232-3238, 2013.

[4] Chucri A Kardous and Peter B Shaw. Evaluation of smartphone sound measurement applications. The Journal of the Acoustical Society of America, 135(4):EL186-EL192, 2014.

[5] Maged N Kamel Boulos, Steve Wheeler, Carlos Tavares, and Ray Jones. How smartphones are changing the face of mobile and participatory healthcare: an overview, with example from ecaalyx. Biomedical engineering online, 10(1):24, 2011. 
[6] Janine Morley, Kelly Widdicks, and Mike Hazas. Digitalisation, energy and data demand: The impact of internet traffic on overall and peak electricity consumption. Energy Research ES Social Science, 38:128-137, 2018.

[7] L Saad. Nearly half of smartphone users can't imagine life without it. gallup, 2015.

[8] Zachary Davies Boren. There are officially more mobile devices than people in the world. The Independent, 7, 2014.

[9] J Anderson. Research in supersonic flight and the breaking of the sound barrier. Retrieved September, 30:2009, 2001.

[10] SM Yahya. Fundamentals of compressible flow: SI units with aircraft and rocket propulsion. New Age International, 2003.

[11] Ashish Garg. Aerodynamics. GATE Aerospace Forum Educational Services, 2015.

[12] Edward M Greitzer, Choon Sooi Tan, and Martin B Graf. Internal flow: concepts and applications, volume 3. Cambridge University Press, 2007.

[13] Shivam Singhal and Ashish Garg. Fluid dynamics: An android application. http:// gateaerospaceforum.com/download.php, December 2014.

[14] John David Anderson Jr. Fundamentals of aerodynamics. Tata McGraw-Hill Education, 2010.

[15] Genick Bar-Meir. Gas dynamics tables. Potto Project, 2007.

[16] William J. Devenport. Compressible aerodynamics calculator 3.0. http://www.dept.aoe. vt.edu/ devenpor/aoe3114/calc.html, February 2008. 